\title{
Perception of healthcare practitioners of the importance of routine oral-cancer screening
}

\begin{abstract}
Introduction: In recent times, both the incidence of and death rate from oral cancers (OC) have decreased significantly due to preventive measures, early detection techniques and enhanced knowledge of clinical signs and symptoms of the disease. Oral cancer is the 11th most common cancer in the world with approximately 389,000 new cases per year. In Saudi Arabia, it is the 3rd most diagnosed malignancy. A direct relationship between tobacco use and oral cancer has been established. The Saudi smoker population has been observed to consume over 80 million cigarettes annually. Smoking among the Saudi general population has reached 52.3\%, which makes Saudi smokers high-risk patients of oral cancer. As tumors of the oral cavity arise on an accessible and partially visible epithelial surface, they should, in principle, be diagnosed early. However, many of these tumors are diagnosed only when they are at an advanced TNM stage, typically in late terminal stages.
\end{abstract}

Purpose: This survey study determines the efficiency, effectiveness and awareness of dental practitioners in Riyadh of oral cancer, incorporation of routine screening for oral cancer in daily dental practice. With the increased rates of $\mathrm{OC}$ diagnosis in late stages, this study was designed to evaluate the healthcare practitioner's knowledge towards OC and its causes in addition to preventive routine screening of OC.

Materials and methods: A survey of randomly selected dental practitioners in public and private clinics was used. Practitioners outside Riyadh city and students were excluded. Results were analyzed using SPSS version-19 software. Chi-square tests (Pearson) were used for analytical statistical figures and probability values; descriptive figures such as graphs were also used.

Results: A total number of dentists contacted were 713 of which 500 responded. Around $50 \%$ of the respondents missed out on examining patients' for oral mucosal surfaces fully, especially the ventral and lateral surfaces of the tongue and the floor of the mouth.

Conclusion: Based on the results it was concluded that most dentists practicing in Riyadh fail to examine the patients routinely. Thus, it is advised that educating programs and awareness campaigns are to be implemented immediately, along with the facilitation of referral between dental departments and clinics.

Keywords: early detection, oral-cancer, prevention, routine screening, death rate, white females, black males
Volume 2 Issue 4 - 2015

\author{
Majd Hasanin,' Nasser Al Asseri, ${ }^{2}$ Jawaher \\ Al Attiyah, ${ }^{3}$ Ahmad Assari, ${ }^{4}$ Alwaleed \\ Abushanan $^{5}$ \\ 'Orthodontic Resident at the University at Buffalo, USA \\ ${ }^{2}$ Oral Maxillofacial Surgery Consultant, OMFS Department, \\ Military Hospital, Saudi Arabia \\ ${ }^{3}$ General Dentist, Saudi Arabia \\ ${ }^{4}$ Demonstrator in Oral and Maxillofacial Surgery and Diagnostic \\ Sciences Department, Riyadh Colleges of dentistry and \\ Pharmacy, Saudi Arabia \\ ${ }^{5}$ Pediatric Dental Resident at the University of British Columbia, \\ Canada
}

Correspondence: Majd Hasanin, Department of Orthodontics, University at Buffalo, USA, Tel I-7I6-322-8057,

Email mshassanain@gmail.com

Received: March 13, 2015 | Published: June 23, 2015
Abbreviations: OC, oral cancers; GPs, general practitioners; SDS, saudi dental society; HCP, health care practitioners

\section{Introduction}

In recent times, both the incidence of and death rate from oral cancers have decreased significantly because of public preventive measures, early detection techniques and enhanced knowledge of clinical signs and symptoms of the disease. Oral cancer is the eleventh most common cancer in the world, with approximately 389,000 new oral cancer cases per year, two-thirds of which occur in developing countries. ${ }^{1,2}$ The American Cancer Society's key statistics has estimated that about 34,300 new cases of oral cavity and oropharynx are diagnosed in the United States annually, eventually leading to approximately 7,000 deaths, which accounts for one-third of the overall cases. ${ }^{3}$ In China, it is the fourth highest malignant disease in males and seventh in females. ${ }^{4}$ In Saudi Arabia, oral cancer is the third most common malignancy diagnosed. ${ }^{5}$ The major risk factors for oral cancers are the use of all forms of tobacco products, alcohol consumption, and sunlight in the case of lip cancer. ${ }^{6}$ It has been estimated that tobacco use accounts for more than $90 \%$ of oral cancers. ${ }^{7}$ Other potential risk factors are exposure to certain viruses (such as the human papillomavirus) and use of marijuana. ${ }^{6}$ This shows that there is a direct relationship between the use of tobacco and the occurrence of oral cancers. The Saudi population has been observed to consume over 80 million cigarettes annually, ${ }^{8}$ and prevalence of smoking amongst them has reached up to $52.3 \%,{ }^{9}$ which ranks them as high-risk patients, in addition to the role of already banned 'Shamma', which is a quid used by many local inhabitants. ${ }^{10}$ Preliminary clinical observations indicated that long-term users of 'shamma' may develop oral cancer. ${ }^{11}$

As tumors of the oral cavity arise on an accessible and partially visible epithelial surface, they should, in principle, be diagnosed early. Unfortunately oral cancer is typically discovered in late terminal stages. ${ }^{3}$ On the other hand, treatment at an early stage may improve survival rate to above $80 \%{ }^{12}$ Although these cancers are easily detectable, the proportion of oral cancers diagnosed in early stage ranges from a low of $26 \%$ among Black males to a high of $48 \%$ among White females. ${ }^{13}$ The knowledge of oral cancer risk factors has 
been reported to be a predictor for having had or being aware of the existence of an oral cancer examination, ${ }^{13}$ which is why; awareness is the key to prevention. By following the three objectives related to oral cancer mentioned in Healthy People 2010, ${ }^{6}$ this will be achieved.

\section{Healthy people 20 I 0 objectives}

Objective 3-6 reduce the oropharyngeal cancer death rate. Objective 21-6 Increase the proportion of oral and pharyngeal cancers detected at the earliest stage. Objective 21-7 Increase the proportion of adults who, in the past 12 months, report having had an examination to detect oral and pharyngeal cancers. The purpose of the study is to determine the efficiency and effectiveness of healthcare practitioners incorporating routine screening for oral cancer in daily dental practice. The investigators hypothesize that there is a lack of awareness towards oral-cancer and this hypothesis was made as a result of late oralcancer diagnosis. Therefore, the aim is targeted towards increasing the awareness of dental practitioners and highlighting the significance of oral-cancer screening as a preventive measure hoping to eventually decrease the morbidity rates caused from this disease.

\section{Materials and methods}

\section{Study design and sample}

A questionnaire was composed of close-ended questions and was randomly distributed among dental practitioners practicing in both licensed private and public dental clinics that are registered in the directory of Ministry of Health in Riyadh city. The targeted practitioners included Dental Interns, General Practitioners (GPs), Specialist and Consultants. The questionnaire was approved by the Saudi Dental Society (SDS)-Research Committee. Practitioners outside Riyadh city and students were excluded. Seven hundred and thirteen dentists were invited to participate, of those only five hundred responded. The questionnaire was specifically designed for this survey in order to assess the knowledge, practice behavior and awareness of the dental practitioners towards oral cancer and its early detection. The items included in the questionnaire were divided into several sections that aimed to assess the knowledge of the practitioner towards the risk factors for oral cancer, another section was to assess the practice behavior which constitutes the main part of the questionnaire, where all the intraoral surfaces were separately posted in words and pictures and the dentist was asked whether he/she examines those surfaces for walk-in patients on each visit. The surfaces that were included in the survey were: Hard and soft mucosal surfaces of the oral cavity, lips and the extra-oral lymph nodes.

\section{Data collection}

The survey was conducted through two methods: Paper and Online and was collected accordingly.

\section{Data analysis}

Results were analyzed using SPSS version-19 software. Chisquare tests (Pearson) were used for analytical statistical figures and probability values; descriptive figures such as graphs were also used.

\section{Results}

Five hundred of the seven hundred thirteen dentists contacted (70\%) responded; of those responded, 214 (42.8\%) were found to always examine the surfaces routinely. Comparing the behavior toward examining the different surfaces, it was found that the gingival was the highest surface examined followed by the lips. And the least examined surfaces were the lateral and ventral surfaces of the tongue. No significance in these findings was found between male and females practitioners (Table 1). When assessing the awareness of the practitioners, $3 \%$ only take panoramic views for first visit patients. Whereas $15 \%$ ask the patients about alcohol usage (Figure 1), which is understandable due to that fact, that consumption of alcohol is illegal in Saudi Arabia.

When comparing the behaviors over the years of experience, the significant difference was only found during examination of the gingiva and the tongue's ventral surface. Although, there was an obvious increase in the number of practitioners who routinely examine the different surfaces routinely as their years of experience increase (Table 2) the behavior towards the routine examination was compared between practitioners with different degrees of education, including dental interns, general practitioners, specialists and consultants. The results showed significant differences between these groups with the practitioners with higher degree of education being more aware about the importance of the surfaces routine examination (Table 3). When the location of the practice between participants was investigated, the results showed that private practitioners are giving more attention to examining surfaces. The difference however, was of statistical significance with respect to the buccal mucosa, tongue's lateral surfaces and hard palate (Table 4).

Table I Behavior towards surfaces routinely examined among genders

\begin{tabular}{llll}
\hline Questions/Variable: Gender & $\begin{array}{l}\text { Male } \mathbf{n}=\mathbf{2 8 7} \text {. (Who } \\
\text { Answered "Yes") }\end{array}$ & $\begin{array}{l}\text { Female } \mathbf{n}=\mathbf{2} \text { | 3. (Who Answered } \\
\text { "Yes") }\end{array}$ & P-value \\
\hline $\begin{array}{l}\text { I. Do you examine: Extra-oral lymph } \\
\text { nodes }\end{array}$ & $140(48.8 \%)$ & $106(49.8 \%)$ & 0.828 \\
2. Lips & $230(80.1 \%)$ & $177(83.1 \%)$ & 0.4 \\
3. Gingival & $263(91.6 \%)$ & $203(95.3 \%)$ & 0.107 \\
4. U \& L Labial Mucosa & $188(65.5 \%)$ & $133(62.4 \%)$ & 0.48 \\
5. Rt. \& Lf. Buccal Mucosa & $191(66.6 \%)$ & $134(62.9 \%)$ & 0.399 \\
6. Tongue's Dorsum Surf & $206(71.8 \%)$ & $169(79.3 \%)$ & 0.053 \\
7.Tongue's Lateral Surf & $127(44.3 \%)$ & $88(41.3 \%)$ & 0.512 \\
8. Tongue's Ventral Surf & $116(40.4 \%)$ & $82(38.5 \%)$ & 0.664 \\
9. Floor of the Mouth & $132(46.0 \%)$ & $97(45.5 \%)$ & 0.92 \\
I0. Hard Palate & $176(61.3 \%)$ & $132(62.0 \%)$ & 0.883 \\
\hline
\end{tabular}

*Differences significant at $\mathrm{p}<0.05$ 


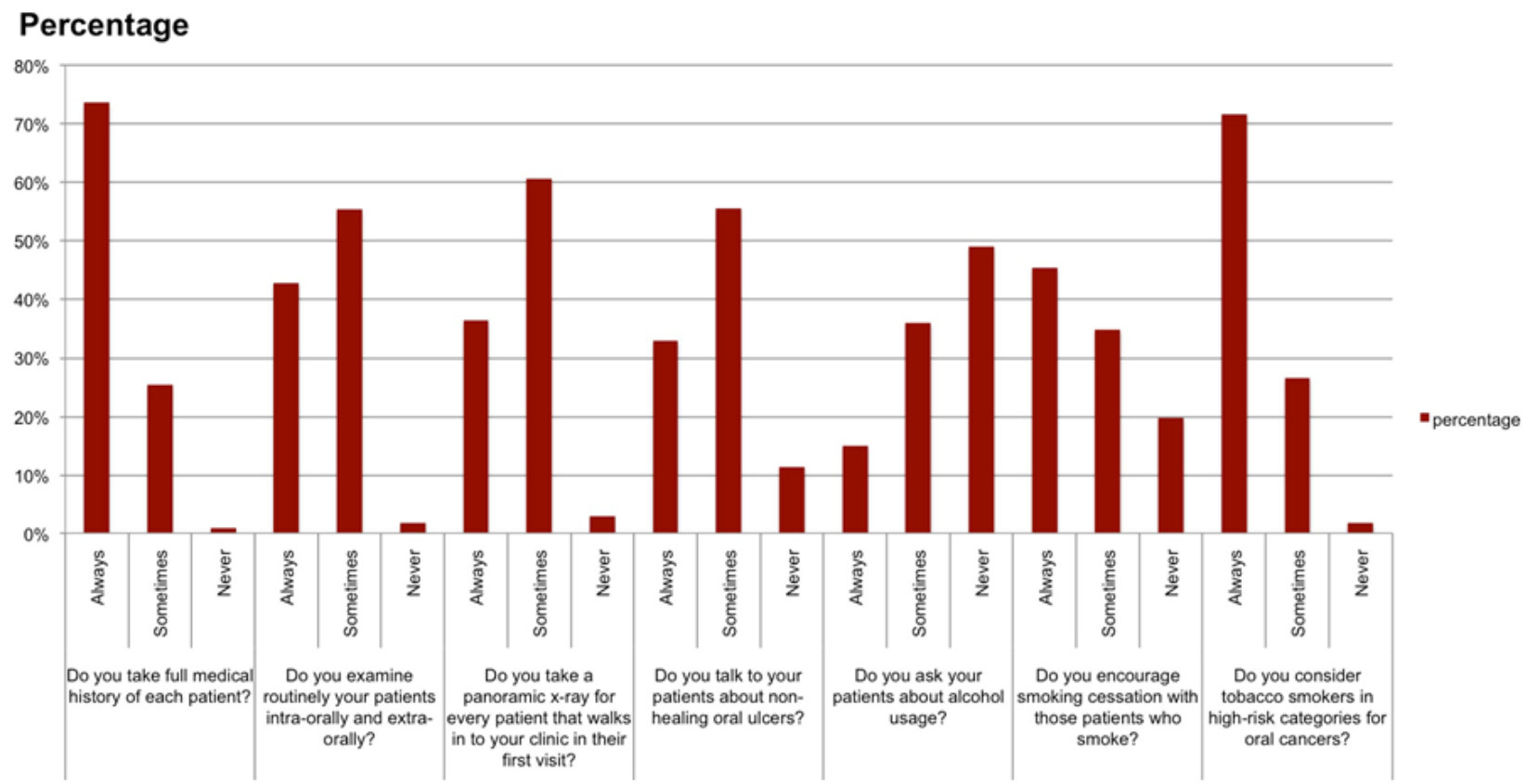

Figure I Response of "awareness-related" questions.

Table 2 Behavior changes in practitioners with different years of experience

\begin{tabular}{|c|c|c|c|c|}
\hline Questions/Variable:Years Of Experience $(n=500)$ & $0-5$ Years & 6-10 Years & $>$ I I Years & p-value \\
\hline I. Do you examine: Extra-oral lymph nodes & $82(43.4 \%)$ & $61(50.0 \%)$ & $103(54.5 \%)$ & 0.095 \\
\hline 2. Lips & $158(83.6 \%)$ & $99(81.1 \%)$ & I 50 (79.4\%) & 0.57 \\
\hline 3. Gingiva & $184(97.4 \%)$ & $112(91.8 \%)$ & $170(89.9 \%)$ & $.013^{*}$ \\
\hline 4. U \& L Labial Mucosa & $115(60.8 \%)$ & $74(60.7 \%)$ & $132(69.8 \%)$ & 0.122 \\
\hline 5. Rt. \& Lf. Buccal Mucosa & $123(65.1 \%)$ & $73(59.8 \%)$ & $129(68.3 \%)$ & 0.315 \\
\hline 6. Tongue's Dorsum Surf & $|4|(74.6 \%)$ & $96(78.7 \%)$ & I $38(73.0 \%)$ & 0.523 \\
\hline 7.Tongue's Lateral Surf & 75 (39.7\%) & $46(37.7 \%)$ & $94(49.7 \%)$ & 0.057 \\
\hline 8. Tongue's Ventral Surf & $63(33.3 \%)$ & $42(34.4 \%)$ & $93(49.2 \%)$ & $.003 *$ \\
\hline 9. Floor of the Mouth & $189(37.8 \%)$ & $122(24.4)$ & $189(37.8 \%)$ & 0.213 \\
\hline 10. Hard Palate & $120(63.5 \%)$ & $72(59.0 \%)$ & $116(61.4 \%)$ & 0.728 \\
\hline
\end{tabular}

*Differences significant at $p<0.05$

Table 3 Behavior changes in practitioners with different degree of education

\begin{tabular}{|c|c|c|c|c|c|}
\hline Questions/Variable: Degree $(n=500)$ & Interns & GP & Specialists & Consultants & p-value \\
\hline I. Do you examine: Extra-oral lymph nodes & $10(26.3 \%)$ & $125(48.8 \%)$ & $68(52.3 \%)$ & $4 \mathrm{I}(56.9 \%)$ & $.017^{*}$ \\
\hline 2. Lips & $33(86.8 \%)$ & 201 (78.5\%) & $107(82.3 \%)$ & $63(87.5 \%)$ & 0.262 \\
\hline 3. Gingiva & $36(94.8 \%)$ & $232(90.6 \%)$ & $127(97.6 \%)$ & 67 (93\%) & 0.075 \\
\hline 4. U \& L Labial Mucosa & $25(65.8 \%)$ & 142 (55.4\%) & $93(71.5 \%)$ & $59(82 \%)$ & $.000 *$ \\
\hline 5. Rt. \& Lf. Buccal Mucosa & $30(78.9 \%)$ & I4I (55\%) & $93(71.5 \%)$ & $59(82 \%)$ & $.000 *$ \\
\hline 6. Tongue's Dorsum Surf & $31(81.6 \%)$ & 191 (74.6\%) & $96(73.8 \%)$ & $55(76.3 \%)$ & 0.787 \\
\hline 7.Tongue's Lateral Surf & $19(50 \%)$ & $92(35.9 \%)$ & $63(48.4 \%)$ & $39(54.1 \%)$ & $.011 *$ \\
\hline 8. Tongue's Ventral Surf & II (28.9\%) & $83(32.4 \%)$ & $60(46.1 \%)$ & $42(58.3 \%)$ & $.000 *$ \\
\hline 9. Floor of the Mouth & $19(50 \%)$ & $96(37.5 \%)$ & $66(50.7 \%)$ & $46(63.9 \%)$ & $.000 *$ \\
\hline 10. Hard Palate & 27 (7I\%) & 130 (50.9\%) & 92 (70.8\%) & 57 (79.1\%) & $.000 *$ \\
\hline
\end{tabular}

*Differences significant at $\mathrm{p}<0.05$

Citation: Hasanin M, Asseri NA, Attiyah JA, et al. Perception of healthcare practitioners of the importance of routine oral-cancer screening.J Dent Health Oral Disord Ther. 20 I5;2(4): I47-I5I. DOI: I0.15406/jdhodt.20I5.02.00060 
Table 4 Comparing examination behaviors of private sector practitioners' vs governmental practitioners

\begin{tabular}{llll}
\hline Questions/Variable: Location (n=500) & $\begin{array}{l}\text { Private } \mathbf{n = 3 ~ I ~ 3 . ~ ( w h o ~} \\
\text { answered "Yes") }\end{array}$ & $\begin{array}{l}\text { Government } \mathbf{n = 1 7 7 . ~ ( w h o ~} \\
\text { answered “Yes") }\end{array}$ & P-value \\
\hline I. Do you examine: Extra-oral lymph nodes & $153(62.2 \%)$ & $87(35.4 \%)$ & 0.787 \\
2. Lips & $249(61.2 \%)$ & $150(36.9 \%)$ & 0.363 \\
3. Gingiva & $289(62.0 \%)$ & $167(35.8 \%)$ & 0.479 \\
4.U \& L Labial Mucosa & $196(61.1 \%)$ & $118(36.8 \%)$ & 0.62 \\
5. Rt. \& Lf. Buccal Mucosa & $187(57.5 \%)$ & $131(40.3 \%)$ & $.006 *$ \\
6. Tongue's Dorsum Surf & $232(61.9 \%)$ & $137(36.5 \%)$ & 0.392 \\
7.Tongue's Lateral Surf & $122(56.7 \%)$ & $86(40.0 \%)$ & $.026 *$ \\
8.Tongue's Ventral Surf & $112(56.6 \%)$ & $80(40.4 \%)$ & $0.05 \mid$ \\
9. Floor of the Mouth & $138(60.3 \%)$ & $85(37.1 \%)$ & 0.465 \\
I0. Hard Palate & $178(57.8 \%)$ & $124(40.3 \%)$ & $.016 *$ \\
\hline
\end{tabular}

*Differences significant at $\mathrm{p}<0.05$

\section{Discussion}

The purpose of the study is to determine the efficiency and effectiveness of healthcare practitioners incorporating routine screening for oral cancer in daily dental practice. The investigators hypothesize that there is a lack of awareness towards oral-cancer and this hypothesis was made as a result of late oral-cancer diagnosis. Therefore, the aim is targeted towards increasing the awareness of dental practitioners and highlighting the significance of oral-cancer screening as a preventive measure hoping to eventually decrease the morbidity rates caused from this disease. Although the highest number of "yes" answers were those by the GPs, it was dependent upon the fact that the questionnaire when randomly distributed, most of the qualifications met were GPs; highlighting the fact that there is a shortage in specialists and consultants in comparison to the number of general practitioners. Furthermore another explanation that could elaborate such results is the enthusiastic drive in young practitioners, specifically when they experience their first job after graduation. Moreover practitioners working in the private sector seem to be paying more attention towards routine oral cancer screening which could be explained by their cautious approach in fear of being sued by patients especially that this practice represents them and it is how they build their reputation.

It has to be noted that most dentists practicing in Riyadh failed to examine the patients routinely for evidence of significant mucosal changes. This was reflected by their lack of sufficient examination of vital surfaces such as: the tongues' lateral and ventral surfaces and the floor of the mouth. This should not be so, in the light of the evidence, ${ }^{3}$ the most common site for OC is the lateral aspect of the tongue and the floor of the mouth. The results in our study also showed that patients were not being routinely asked (\%15) about smoking and alcohol abuse even though the later is forbidden in the Saudi Arabian environment, both are strongly related to OC. ${ }^{14}$ It has to be mentioned that oral cancer is associated with high mortality and morbidity rates, largely as a result of late diagnosis, ${ }^{15}$ thus early diagnosis and treatment of cancer are essential to achieving a good prognosis. ${ }^{16}$ Evidence in the literature continues to confirm that in developing countries, OSCC shows high morbidity and mortality.,17 However, many of these tumors are diagnosed only when they are at an advanced stage, and the 5-year relative survival rate from the time of diagnosis is around $40 \%,{ }^{18}$ and the financial cost to treat and rehabilitate patients with this devastating disease is estimated to be about 2 billion dollars. ${ }^{19}$

Both patient and clinician factors play a role in this delayed diagnosis, and its detection in its early stages has become a significant matter in the medical field in the management of the disease.$^{20}$ Efforts have been made towards understanding the causes of oral cancer and assessing proper interventions, but yet, prevention still requires much more attention. Furthermore health care practitioners (HCP) have a major responsibility in the prevention and early detection of oral cancer, which is why an adequate training in this area will positively enhance the practitioner's accountability towards their patients. ${ }^{1}$ Dentists can detect it earlier than any other practitioner, ${ }^{21}$ meaning clinicians should think of prevention in two ways: early detection; and the opportunity to identify and treat pre-malignant lesions. A more routinized and adequate examination of the mucosal surfaces at risk will go a long way in the early detection of OSCC. What was evident in the literature reports on OSCC was the absence of standardized guidelines of oral mucosa examination that are universally practiced. There is however a consensus that routine screenings were effective at $60 \%$ sensitivity and $90 \%$ specificity and most importantly that visual inspection is very effective. ${ }^{22-24}$ It appears that the current knowledge and training in oral cancer prevention and early detection among HCPs in Saudi Arabia is not up to the desired level. Accordingly, it is suggested that it should be given the necessary attention when establishing new continuous education strategies in Saudi Arabia, ${ }^{1}$ where dentists need to play a role in establishing a new paradigm for oral cancer control, ${ }^{15}$ as they are in a position to advise and help protect their patients, staff, colleagues, families and acquaintances..$^{25}$ The study reflected the health care standards in Riyadh city, the largest city in Saudi Arabia which consists of a large population of dentists that might reflect the rest of the country. Furthermore the study focused on different levels of education related to dentists, to be more detailed and gave a bigger understanding of the routine oral-cancer screening. However a more broad study covering dentists in different parts of Saudi Arabia is recommended to have a better representation of the health care standards carried. Moreover as the study was conducted through a survey it might have the potential bias of the respondents. Also the prevalence of oral-cancer in other regions might be higher that could lead to practitioners responding differently to them as they are more exposed to such cases. 


\section{Conclusion}

Based on the results it was concluded that most dentists practicing in Riyadh failed to examine the patients routinely for evidence of significant mucosal changes. Oral cancer screening is not an expensive tool nor is it time consuming and if practiced by dental practitioners routinely, it will prove to be very effective and lifesaving by detecting OC early. To achieve such lifesaving measures and raise the awareness in the community, it is advocated that the following recommendations take place:

1. The implementation of training programs for healthcare practitioners aimed at the prevention and early detection of oral cancer.

2. Emphasize on patient awareness of oral cancer, as a preventable disease should be practiced during routine dental check-ups.

3. As there are no clear standardized guidelines for routine oral cancer screening, it is advised that a universally understood and accepted protocol for routine examination by the World Health Organization is issued.

The authors also recommend that further studies should take place, covering different parts of Saudi Arabia, as well as conducting studies related to the perception of patients and how aware they are to oralcancer and the importance of early diagnosis, as it is very important to have patients self-screen themselves in order for the dentist to early diagnose oral-cancer.

\section{Acknowledgment}

This research was done in dedication to Sameer Hasanin (may god rest his soul in peace) who passed away 17 years ago due to oral cancer. The authors would like to thank Prof. H.A. Mosadomifor his continuous support. Dr. Nasser Muflehi for assisting with the data analysis, Dr. Mohammad Obeidafor facilitating communication with other parties and all the dental practitioners who took the time and participated in the research and above all my mother Dr. ArwaObeidat.

\section{Conflicts of interest}

The author declares that there are no conflicts of interest.

\section{Funding}

None

\section{References}

1. Jaber $\mathrm{L}$, Shaban $\mathrm{S}$, Hariri $\mathrm{D}$, et al. Perceptions of healthcare practitioners in Saudi Arabia regarding their training. Int $J$ Health Care Qual Assur. 2011;24(1):8-18.

2. Sankaranarayanan R, Fernandez Garrote L, Lence Anta J, et al. Visual inspection in oral cancer screening in Cuba: a case-control study. Oral Oncol. 2002;38(2):131-136.

3. What are the key statistics about oral cavity and oropharyngeal cancers? The American Cancer Society. 2014.

4. Kao SY, Chu YW, Chen YW, et al. Detection and screening of oral cancer and pre-cancerous lesions. J Chin Med Assoc. 2009;72(5):227-233.
5. Al-Balawi SA, Nwoku AL. Management of oral cancer in a tertiary care hospital. Saudi Med J. 2002;23(2):156-159.

6. Canto MT, Drury TF, Horowitz AM. Use of skin and oral cancer examinations in the United States, 1998. Prev Med. 2003;37(3):278-282.

7. Tobacco and Oral Health; Action on Smoking and Health. ASH Research Report. 2012.

8. Dammam, Asharq Al-Awsat. Saudis Consume Over 80 Million Cigarettes Annually. 2006.

9. Bassiony MM. Smoking in Saudi Arabia. Saudi Med J. 2009;30(7):876881.

10. Yousef A, Hashash M. Common features and surgical interference in a prevalent oral cancer in Saudi. J Laryngol Otol. 1983;97(9):837-843.

11. Hannan MA, el-Yazigi A, Paul M, et al. Genotoxicity of 'shamma', a chewing material suspected of causing oral cancer in Saudi Arabia. Mutat Res. 1986;169(1-2):41-46.

12. Speight PM, Morgan PR. The natural history and pathology of oral cancer and precancer. Community Dent Health. 1993;10(1):31-41.

13. Gajendra S, Cruz GD, Kumar JV. Oral cancer prevention and early detection: knowledge, practices, and opinions of oral health care providers in New York state. J Cancer Educ. 2006;21(3):157-162.

14. Opportunistic Oral Cancer Screening, A management strategy for dental practice. BDA Occasional Paper. 2000;6:1-36.

15. Rosin MP, Poh CF, Elwood JM, et al. New hope for an oral cancer solution: together we can make a difference. $J$ Can Dent Assoc. 2008;74(3):261266.

16. Onizawa K, Nishihara K, Yamagata K, et al. Factors associated with diagnostic delay of oral squamous cell carcinoma. Oral Oncol. 2003;39(8):781-788.

17. Greene SA. Spotting Oral Cancer. 2009.

18. Elwood JM, Gallagher RP. Factors influencing early diagnosis of cancer of the oral cavity. CMAJ. 1985;133(7):651-656.

19. Reisine LD. Social, psychological, and economic impacts of oral conditions and treatments. In: Cohen LK \& Gift HC, editors. Disease Prevention and Oral Health Promotion Copenhagen, Denmark, UK; 1995.

20. Pitiphat W, Diehl SR, Laskaris G, et al. Factors associated with delay in the diagnosis of oral cancer. $J$ Dent Res. 2002;81(3):192-197.

21. Holmes JD, Dierks EJ, Homer LD, et al. Is detection of oral and oropharyngeal squamous cancer by a dental health care provider associated with a lower stage at diagnosis? J Oral Maxillofac Surg. 2003;61(3):285-291.

22. Stahl S, Meskin LH, Brown LJ. The American Dental Association's oral cancer campaign: the impact on consumers and dentists. J Am Dent Assoc. 2004;135(9):1261-1267.

23. Guideline for the early detection of oral cancer in British Columbia. J Can Dent Assoc. 2008;74(3):245.

24. Ramadas K, Sankaranarayanan R, Jacob BJ, et al.Interim results from a cluster randomized controlled oral cancer screening trial in Kerala, India. Oral Oncol. 2003;39(6):580-588.

25. Scully C, Newman L, Bagan JV. The role of the dental team in preventing and diagnosing cancer: 2. Oral cancer risk factors. Dent Update. 2005;32(5):261-270. 